\title{
Intra-Aneurysmal Pressure and Flow Changes Induced by Flow Diverters: Relation to Aneurysm Size and Shape
}

\author{
I. Larrabide, M.L. Aguilar, H.G. Morales, A.J. Geers, Z. Kulcsár, D. Rüfenacht, and A.F. Frangi
}

\begin{abstract}
BACKGROUND AND PURPOSE: Effects of blood flow modification by flow diverters are observed to lead often to aneurysm thrombosis and reverse remodeling. For this process, to further understand the potential roles of intra-aneurysmal blood pressure changes and aneurysm morphologies, 23 patients were studied by numeric simulation.
\end{abstract}

MATERIALS AND METHODS: 3D imaging of aneurysms of different sizes and shapes, all located at the supraclinoid segment of the ICA $(n=23)$, was prepared for CFD simulations. Hemodynamic variables were calculated for conditions before and after virtual FD implantation, reconstituting a vessel wall scaffold across the aneurysm neck. WSS, velocity, residence time, turnover time, and intra-aneurysmal pressure were assessed statistically.

RESULTS: After placement of FDs, significant reductions inside the aneurysm were observed for most hemodynamic variables $(P<.01)$ except mean intra-aneurysmal pressures. For minimum/maximum intra-aneurysmal pressure values, small but significant changes were found; however, they were considered too small to be of relevance.

CONCLUSIONS: Calculations in 23 cases did not reveal significant intra-aneurysmal mean or peak pressure changes, indicating a minor role of pressure changes in the rare event of secondary ruptures after FD use. Other hemodynamic variables (WSS and velocity) exhibited more significant changes, indicating their role in intra-aneurysmal thrombus formation. Size-dependent, significantly higher reduction in WSS $(P=.069)$ and velocity $(P=.013)$ was observed in small aneurysms compared with larger ones. When it came to shape, there were significantly higher reductions in WSS $(P=.055)$ and velocity $(P=.065)$ and a significantly higher increase in turnover time in fusiform aneurysms compared with saccular aneurysms.

ABBREVIATIONS: CFD = computational fluid dynamics; FD = flow diverter; IA $=$ intracranial aneurysm; WSS $=$ wall shear stress

$\mathbf{F}^{\mathrm{i}}$ Ds are stentlike devices designed to redirect the blood flow through the parent vessel by diverting it away from the aneurysm. This effectively reduces the flow into the aneurysm, hereby

Received April 28, 2012; accepted after revision June 25.

From the Networking Biomedical Research Center on Bioengineering (I.L., M.L.A., H.G.M., A.J.G., A.F.F.), Biomaterials and Nanomedicine (CIBER-BBN), Barcelona, Spain; Center for Computational Imaging \& Simulation Technologies in Biomedicine (CISTIB) (I.L., M.L.A., A.J.G., A.F.F.), Universitat Pompeu Fabra, Barcelona, Spain; and Hirslanden Clinic (Z.K., D.R.), Zürich, Switzerland.

Preliminary results previously presented at: Second International Conference on Mathematical and Computational Biomedical Engineering, March 30-April 1, 2011; Washington DC; and Eighth International Interdisciplinary Cerebrovascular Symposium, September 8-11, 2011; Shanghai, China.

Ignacio Larrabide and Martha L. Aguilar contributed equally to this work.

This research has been partially funded by the Industrial and Technological Development Center (CDTI) under the CENIT-CDTEAM and CENIT-cvREMOD programs, the European Commission project @neurIST (IST-2005-027703), and Philips Healthcare.

Please address correspondence to I. Larrabide, MD, Center for Computational Imaging \& Simulation Technologies in Biomedicine, Universitat Pompeu Fabra, Tànger 122-140, 08018 Barcelona, Spain; e-mail: ignacio.larrabide@upf.edu

-- Indicates open access to non-subscribers at www.ajnr.org

http://dx.doi.org/10.3174/ajnr.A3288 stimulating thrombosis and, ultimately, occlusion. ${ }^{1}$ These changes in local blood flow have been previously quantified from angiography. ${ }^{2,3}$ Also, animal experiments and clinical studies have been developed showing the effectiveness of this technology. ${ }^{1,4}$ Besides complete aneurysm occlusions in most cases, longer-term patency and delayed aneurysm rupture have also been reported. $^{5-9}$ Since the introduction of these devices in clinical practice, different studies have been developed to model them and to provide a better understanding of their effect on hemodynamics. ${ }^{10-13}$

Still, there is controversy about the effect of FDs on some aspects, like the changes on intra-aneurysmal pressure. ${ }^{5}$ To our knowledge, this is the largest CFD study on the effect of FDs with aneurysms of different sizes and shapes so far $(n=23)$, capturing the large anatomic variability.

The purpose of this study was 2-fold: first, assessing the effect of FDs on intra-aneurysmal pressure; second, understanding the effect of FDs on reducing hemodynamic variables, depending on the aneurysm size and shape. 


\section{MATERIALS AND METHODS \\ Materials}

All the aneurysms were drawn from the @neurIST data base, ${ }^{14}$ which was collected in different centers and processed by different observers following the same data-processing protocol. All selected aneurysms were located at the supraclinoid segment of the ICA to reduce differences in the parent vessel flow rate due to varying anatomic locations. Anatomic models were obtained from diagnostic 3D rotational angiography images, acquired through an Integris Allura system (Philips Healthcare, Best, the Netherlands) or an Axiom Artis scanner (Siemens, Erlangen, Germany). Voxel sizes in the reconstructed $3 \mathrm{D}$ images ranged from 0.208 to $0.378 \mathrm{~mm}^{3}$.

For an initial selection from a data base of 40 aneurysms, 3 clinicians independently selected the most appropriate endovascular treatment. The criteria used to select the treatment were the following: 1) branching arteries (typically ophthalmic artery or anterior choroidal artery) near the aneurysm that could be occluded by the device were absent, and 2) an aneurysm suboptimal for treatment with coils because of its morphology and/or neck width. A subset of 23 aneurysms was chosen for treatment with FDs by at least 2 clinicians. The remaining aneurysms ${ }^{15}$ were considered by $\geq 2$ clinicians to be more suitable for conventional treatment and were excluded from the study. Eleven aneurysms (47\%) were located at the ophthalmic artery; 9 (38\%), at the posterior communicating artery; and 3 (13\%), at the anterior choroidal artery. Aneurysms sizes ranged between 1.3 and $22 \mathrm{~mm}$. Seventy percent of patients were women. Patient age was $50 \pm 8$ years at the moment of aneurysm discovery.

\section{Methods}

Images were segmented by using a geodesic active regions method, and a 3D model consisting of a triangulated surface mesh was generated. ${ }^{16}$ Triangle removal, hole filling, and volume-preserving optimized Laplacian smoothing were used to remove imperfections in the vascular models. Geometric models of the FDs, consisting of 48 wires of $60-\mu \mathrm{m}$ thickness, were created and deployed in the vascular models by using a Fast Virtual Stent placement method. ${ }^{17}$ After stent deployment, the mean porosity was measured for each case. The mean porosity for the 23 cases was $72.8 \%$ on average with a coefficient of variation of $7.51 \%$ and minimum and maximum mean porosity of $59.5 \%$ and $80.8 \%$, respectively. Volumetric meshes were generated by using the commercial software ICEM CFD, Version 11.0 (ANSYS, Canonsburg, Pennsylvania). These meshes were composed of unstructured tetrahedral and 8-node prism elements. Mesh sizes ranged between 0.4 and 3.4 million elements for the untreated cases and from 2.6 to 15.2 million elements for the treated cases.

Mesh-independent CFD solutions were obtained for meshes with $0.016-\mathrm{mm}$ elements around the stent struts (ie, approximately 12 elements along the perimeter) and maximally with 0.4-mm elements in the lumen. Following the study of Appanaboyina et $\mathrm{al},{ }^{15}$ the portion of the stent lying on the vessel wall was removed, and only the portion of the stent covering the aneurysm neck was considered, to reduce computational time.

Transient CFD simulations were done with CFX, Version 11.0 (ANSYS), which uses a finite volume approach to solve the Na-
vier-Stokes equations. Blood was modeled as an incompressible Newtonian fluid (attenuation $=1066 \mathrm{~kg} / \mathrm{m}^{3}$, viscosity $=0.0035$ $\mathrm{Pa} \cdot \mathrm{s})$. The vessel wall was assumed to be rigid with a no-slip boundary condition. Flow boundary conditions were derived from a validated $1 \mathrm{D}$ mathematic model of the systemic arterial tree. ${ }^{18}$ Flow-rate waveforms were imposed at the inlet; and pressure waveforms, at the outlets. A straight inlet extension was added to the vascular model, and a flat velocity profile was imposed at the end of the extension. The combination of the extension and the vascular district considered causes that the effects of the imposed velocity profile vanish at the location of the aneurysm. ${ }^{19}$ The flow-rate waveform imposed at the inlet and the location of the inlet were equivalent for all the models, corresponding to the cervical or petrous segment of the ICA, depending on the vessel portion visible from the corresponding $3 \mathrm{D}$ rotational angiography image. One cardiac cycle of 0.8 seconds was divided into time-steps of 0.005 seconds, which were previously found to provide a time-step-independent solution for stented aneurysm models. ${ }^{20}$ To reduce initial transients, we computed 3 complete cardiac cycles, and data of the third cardiac cycle were stored and analyzed.

\section{Data Analysis}

The hemodynamic variables studied were the following:

- Time-averaged WSS on the aneurysm wall (wsstasa) and 90th percentile of the maximum WSS (wssta90). WSS plays a key role in arterial wall remodelling and aneurysm evolution. ${ }^{21}$

- Time and spatial-average velocity in the aneurysm (velsata).

- Spatial average of relative residence time in the aneurysm (rrtsa). Relative residence time increases with decreasing WSS and increasing oscillatory shear index, and it identifies regions of flow stagnation near the wall. ${ }^{22}$

- Aneurysm volume turnover time (totime), which may modulate thrombosis. ${ }^{23}$

- Spatial average intra-aneurysmal pressure (pressuresata), maximum (pressuremaxta) and minimum (pressureminta) pressure, and range (pressurergta) averaged with time inside the aneurysm dome. Different studies indicate changes in intraaneurysmal pressure after the placement of FDs. ${ }^{5,24}$

- Flow-pattern change inside the aneurysm: qualitative variable assessed by authors I.L .and Z.K. by using flow streamlines before and after FD treatment. Consensus between the 2 was achieved in all cases. The change in flow pattern was considered important when both a different inflow pattern and a different intra-aneurysmal vortex pattern were observed. If the main flow pattern remained the same and the hemodynamic changes were attributable only to the flow reduction, the change was not considered important.

All variables were considered before and after placement of the FD. The reduction of variable $\mathrm{X}$ was computed as

1)

$$
\mathrm{X}_{\text {reduction }}=100 \% \cdot \frac{\left(\mathrm{X}_{\text {untreated }}-\mathrm{X}_{\text {treated }}\right)}{\mathrm{X}_{\text {untreated }}}
$$

Pressure was always presented in units of millimeters of mercury. A negative reduction indicated an effective increase. For data 


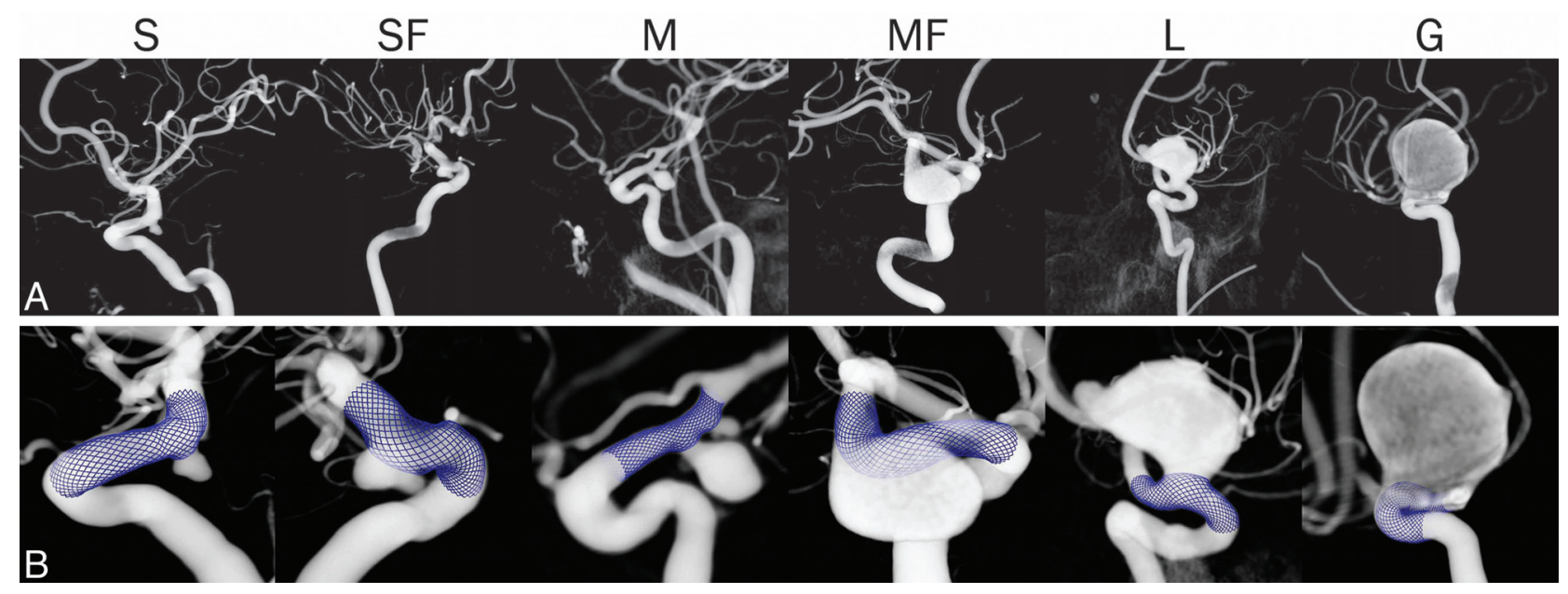

FIG 1. A, 3D rotational angiograms of 5 cases with different sizes and shapes (small, small fusiform, medium, medium fusiform, large, and giant). $B$, Virtually implanted FDs are visualized in blue in the hosting artery.

analysis, the statistical package $\mathrm{R}$ (http://www.r-project.org/) was used. $^{25}$

Aneurysms were classified according to size (small, $<5 \mathrm{~mm}$; medium, 5-10 $\mathrm{mm}$; large, $10-25 \mathrm{~mm}$; or giant, $\geq 25 \mathrm{~mm}$ ) and shape (saccular or fusiform). ${ }^{26}$ Large and giant aneurysms were lumped together in the statistical analysis. Aneurysms with larger neck width than dome depth and width were considered fusiform; the rest were considered saccular. The reduction in hemodynamic variables was compared between groups by using nonparametric tests. Size (3 groups) was assessed with the Kruskal-Wallis 1-way analysis of variance test; and shape (2 groups), with the Wilcoxon rank-sum test.

\section{RESULTS}

\section{Aneurysm Classification in Size and Shape}

The dataset contained 10 small (43\%), 9 medium (39\%), and 4 large/giant (17\%) aneurysms. Sixteen (70\%) aneurysms were saccular, and 7 (30\%) were fusiform. Six selected cases are shown in Fig 1.

\section{Effect of FD on Local Hemodynamics}

Figure 2 shows simulation results at peak systole for 6 cases before and after treatment. After we placed the FDs, the local hemodynamic conditions in the parent vessel and the aneurysm (columns $A, B$, and $C$ ) were changed. Streamlines generated from the proximal end (500 equally distributed at the inlet) and velocity magnitude on a cut plane across the aneurysm are shown on columns $A$ and $B$, respectively. Reduction of velocity can be clearly observed in the velocity magnitude cut planes. This reduction is evident inside the aneurysm in Figure 2. Streamlines show the change in flow patterns, only observed in a few cases (marked with an asterisk). In general, the WSS was reduced at the aneurysm wall after FD placement (Fig 2C). All the hemodynamic variables but pressure showed significant changes (Table $1 ; P<.001$ ) after FD placement, in accordance with previous findings in the literature. $^{10,11,13}$

In 20 of the 23 cases, the flow patterns showed none or minimal changes induced by the placement of the FD. An important change in intra-aneurysmal flow patterns was observed in the other 3 aneurysms, where FDs induced a diffuse inflow and a different spatial configuration of streamlines. The 3 aneurysms were saccular and of different sizes (small, medium, and giant). Flow was found to be shear-driven in the 3 cases with the main jet hitting the aneurysm neck in 2 (small and medium).

\section{Effect of FD on Intra-Aneurysmal Pressure}

Figure 3 shows intra-aneurysmal pressure ranges and means before (blue area and black line) and after (red area and gray line) FD treatment for the 6 cases described previously. The larger changes, on the order of $2 \sim 5 \mathrm{~mm} \mathrm{Hg}$, are observed during diastole. Significant changes $(P<.001)$ were found for pressuremaxta $($ median $=0.34 \mathrm{~mm} \mathrm{Hg})$, pressureminta $($ median $=-0.31 \mathrm{~mm}$ $\mathrm{Hg}$ ), pressurergta (median $=0.61 \mathrm{~mm} \mathrm{Hg}$ ), but not for pressuresata $($ median $=-0.038 \mathrm{~mm} \mathrm{Hg})$. Observed changes were below 2 $\mathrm{mm} \mathrm{Hg}$ for pressuremaxta and pressureminta, and the largest change in pressurergta was $3 \mathrm{~mm} \mathrm{Hg}$ (Fig 3). Negligible changes in all pressure variables ( $<2 \mathrm{~mm} \mathrm{Hg}$, below $3 \%$ of pulse amplitude) were observed for the 3 cases presenting changes in flow patterns.

\section{FD Effect Depending on Aneurysm Size and Shape}

Results for classifications by size and shape are summarized in Table 2. When we classified aneurysms by size, significant differences between groups were found for wsstasa $(P<.1)$, wssta90 $(P<.05)$, and velsata $(P<.05)$. In Fig 4 , we observed that the FD produced a larger reduction on small aneurysms (medians: wsstasa $=68.24 \%$, wssta90 $=65.87 \%$, velsata $=58.04 \%)$ and smaller reductions on large/giant aneurysms (medians: wsstasa = $49.77 \%$, wssta90 $=49.09 \%$, velsata $=34.18 \%)$. Medium-sized aneurysms showed a higher disagreement in hemodynamic variables after FD placement and more changing outcome in terms of hemodynamic variables (range of velsata $=50.56 \%$, wsstasa $=$ 49.40). Although not statistically significant, the same tendency was observed for rrtsa and totime. Comparing only small and large/giant aneurysms, we found statistically significant differences for wsstasa $(P<.05)$, wssta90 $(P<.05)$, velsata $(P<.05)$, and $\operatorname{rrtsa}(P=.055)$ (indicated with brackets on the right of Fig 3$)$.

For the shape classification, as shown in Fig 4, significant dif- 


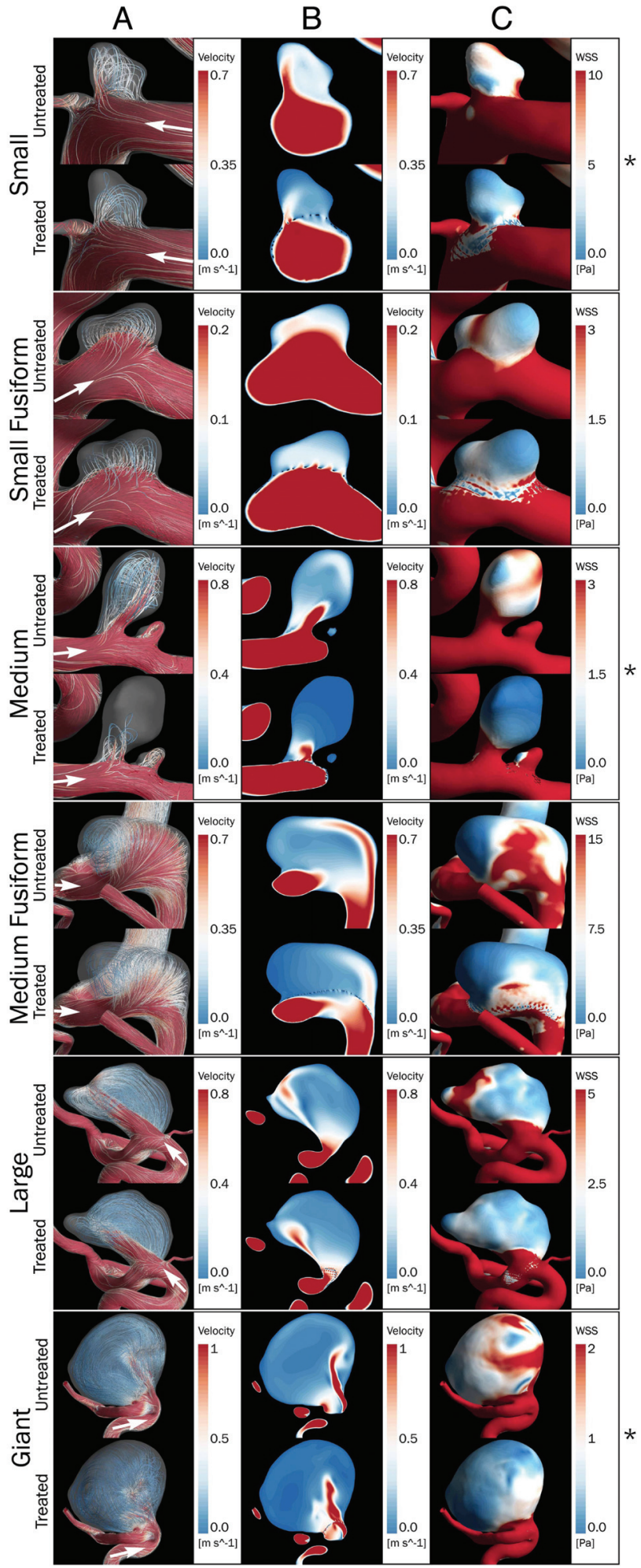

FIG 2. Six of 23 aneurysms studied in this work. For each aneurysm, the first row corresponds to the untreated model, and the second, to the treated one. The different rows correspond to different types (size and shape) of aneurysms. Note streamlines $(A)$, velocity magnitude on a plane across the aneurysm (B), and WSS at peak systole ( $C$ ). White arrows indicate the flow direction. The asterisk on the right indicates cases in which important changes in flow patterns are observed.

ferences were found for wsstasa $(P=.069)$, wssta90 $(P<.05)$, velsata $(P<.05)$, and totime $(P<.05)$ (Table 3$)$.
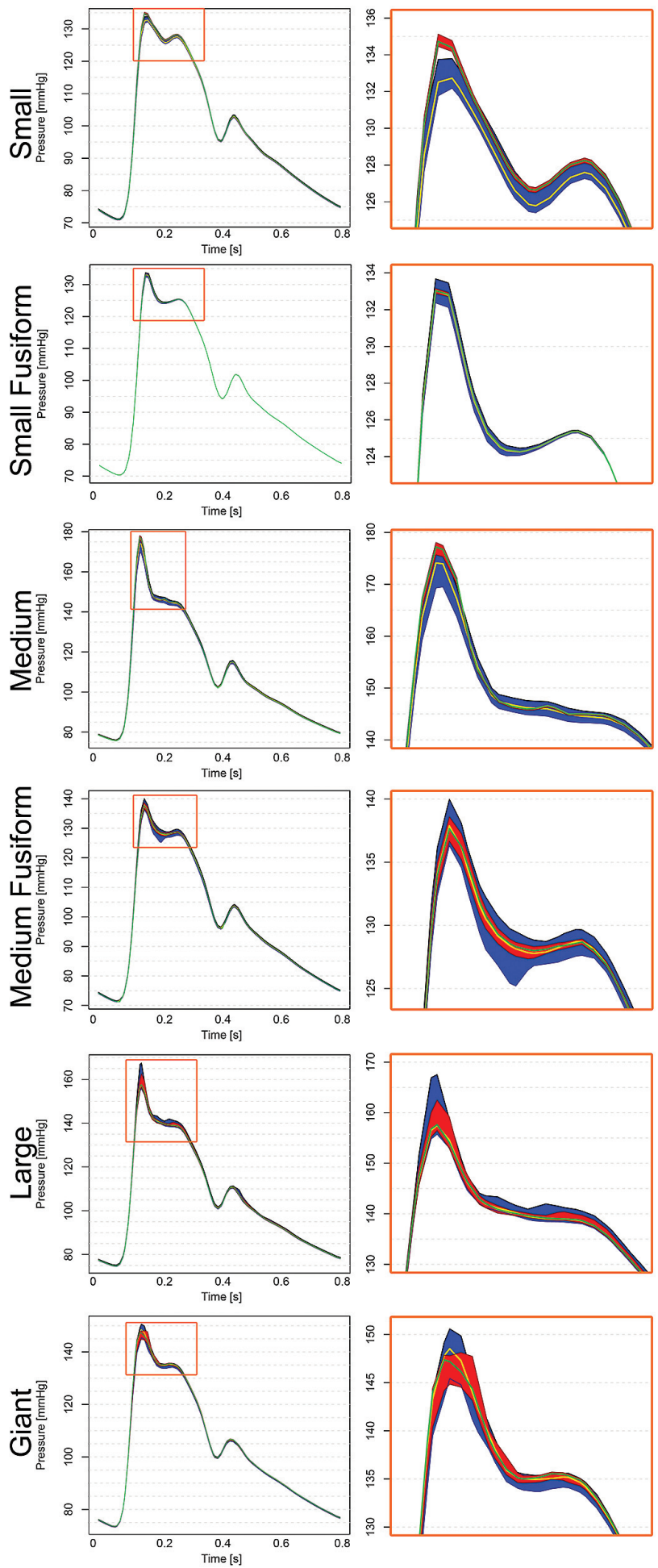

FIG 3. The column on the left shows the pressure range and mean intra-aneurysmal pressure, both indicated before (range in blue and mean with a yellow line) and after (range in red and mean with a green line) treatment for the 6 cases shown above. The column on the right shows a zoom into the plot on peak systole (highlighted in green on the left column).

The reduction was larger on fusiform (medians: wsstasa $=68.53 \%$, wssta90 $=63.81 \%$, velsata $=54.33 \%$, totime $=-113.45 \%$ ) than on saccular aneurysms (median 
Table 1: Hemodynamic variables studied before and after treatment

\begin{tabular}{lccccccc}
\hline & wsstasa & wssta90 & velsata & rrtsa & totime & pressuresata & pressurergta \\
\hline Pvalue $^{\mathrm{a}}$ & $<.001$ & $<.001$ & $<.001$ & $<.001$ & $<.001$ & .482 & $<.001$ \\
Median $^{\mathrm{b}}$ & 55.09 & 58.91 & 52.34 & -185.77 & -69.39 & 78.88 & 0.72 \\
\hline
\end{tabular}

Note:-wsstasa indicates WSS on the aneurysm wall; wssta90, maximum WSS; velsata, time and spatial-average velocity in the aneurysm; rrtsa, spatial average of relative residence time in the aneurysm; totime, aneurysm volume turnover time; pressuressata, spatial average intra-aneurysmal pressure; pressuregta, pressure range.

a $P$ values correspond to the Wilcoxon signed-rank test comparing untreated and treated samples.

${ }^{\mathrm{b}}$ Median reduction after FD placement.

Table 2: $P$ values for size (3 groups, Kruskal-Wallis 1-way analysis of variance by rank test) and shape ( 2 groups, Wilcoxon signed-rank test) versus hemodynamic variables

\begin{tabular}{lccccccc}
\hline & wsstasa & wssta90 & velsata & rrtsa & totime & pressuresata & pressurergta \\
\hline Size & $.069^{\mathrm{a}}$ & $.015^{\mathrm{b}}$ & $.013^{\mathrm{b}}$ & .244 & .195 & .202 \\
Size (S/LG) & $.036^{\mathrm{b}}$ & $.004^{\mathrm{b}}$ & $.002^{\mathrm{b}}$ & $.076^{\mathrm{a}}$ & .142 & .304 \\
Shape & .055 & $.047^{\mathrm{a}}$ & $.065^{\mathrm{a}}$ & 052 & $.047^{\mathrm{b}}$ & \\
\hline
\end{tabular}

Note:-wsstasa indicates WSS on the aneurysm wall; wssta90, maximum WSS; velsata, time and spatial-average velocity in the aneurysm; rrtsa, spatial average of relative residence time in the aneurysm; totime, aneurysm volume turnover time; pressuressata, spatial average intra-aneurysmal pressure; pressuregta, pressure range.

a Significant to the 10th percentile.

b Significant to the 5 th percentile.

c Row size (S/LG) corresponds to Wilcoxon signed-rank test between small and large aneurysms.

wsstasa $=53.43 \%$, wssta90 $=54.19 \%$, velsata $=39.81 \%$, totime $=-54.42 \%)$. Although not statistically significant, the same tendency was observed for rrtsa, for which we observed 2 outliers, corresponding to an increase of $1300 \%$ and $950 \%$ on giant and medium-sized saccular aneurysms, respectively.

None of the pressure-related variables presented statistically significant differences between size or shape groups.

\section{DISCUSSION}

This work presents the largest computational hemodynamics study on IAs treated with FDs so far, to our knowledge. Twenty-three cases located on the supraclinoid segment of the ICA, covering a wide range of different aneurysm sizes and shapes, were considered.

One purpose was to investigate pressure changes induced by the placements of FDs in IAs. FDs showed a significant change in hemodynamic variables like WSS, velocity, and turnover time inside the aneurysm, but not on mean intra-aneurysmal pressure. In general, the effect of the FD was to lower velocity and to reduce the intra-aneurysmal pressure range (decrease of the maximum and increase of the minimum pressures, Figs 3 and 4). These results suggest that irrespective of the aneurysm size and shape, the sole insertion of FDs will not reduce or increase intra-aneurysmal pressure (Table 2). An FD does not affect the static pressure, only the dynamic pressure. The dynamic pressure, which is a function of the velocity, is 2 orders of magnitude smaller than the static pressure, so the change in total pressure can be expected to be $<3 \%$ of the pulse pressure $(<2 \mathrm{~mm} \mathrm{Hg}$ ), as observed. This is in accordance with results by Schneiders et al, ${ }^{24}$ who showed that the placement of FDs does not imply an early reduction of intra-aneurysmal pressure. Recent clinical findings by Cebral et $\mathrm{al}^{5}$ also supported by computational simulations, suggest that placement of FDs leads to an increased intra-aneurysmal pressure. Such increases were not found for the population studied here. We found no evidence suggesting that FDs induce a pressure change that might, by itself, induce IA rupture.

The second purpose of this study was to understand the effect of FDs on reducing hemodynamic variables, depending on the aneurysm size and shape. With respect to size, statistical tests showed that the reduction of WSS and velocity is significantly larger for small aneurysms. In such aneurysms, the flow in the parent vessel and in the aneurysm is closely related. As an aneurysm becomes larger, this relation is weaker. Therefore, placing an FD on the parent vessel has a higher effect on the flow of small aneurysms than on large/giant ones in relative terms. This difference becomes clear from Table 2. We noticed that differences in residence time are also significant in such cases.

Classification by shape showed significant differences for high WSS, velocity, and turnover time. The reduction was found to be more pronounced in fusiform aneurysms. Similar to the case of large/giant aneurysms, the relation between the flow in the aneurysm and in the parent vessel is weak; therefore, the relative effect of FDs on reducing velocity was less in saccular than in fusiform aneurysms. Although the turnover time increased after flow diversion, especially in fusiform aneurysms, the near-wall residence time (rrtsa) was not significantly different between groups. Therefore, from the point of view of residence time, there is no evidence showing that aneurysms of different shape (ie, saccular or fusiform) have a different chance of near-wall flow stagnation.

Nonsignificant correlations were found between the porosity of the FDs and the hemodynamic variable reduction. Still, hemodynamic changes might be related to porosity in a more complex way involving other characteristics of the vasculature (location of the aneurysm, position with respect to the parent vessel, local curvature and torsion of the vessel, and so forth) that might have an impact on the FD porosity and will require further investigation.

Several studies have investigated the effect of FDs on intraaneurysmal hemodynamics. ${ }^{5,13}$ Our results confirm previous findings for all measured variables. ${ }^{10}$ The process of progressive aneurysm occlusion after FD treatment depends on several factors, including intra-aneurysmal hemodynamic changes, which are mainly reflected in pressure-range reduction, mean velocity reduction, WSS reduction, and increase of residence and turnover time. ${ }^{23}$ We noticed that hemodynamic changes do not necessarily imply a change in flow patterns, which were only observed in a few cases (3 of 23). Low-flow velocity and reduced WSS are both important factors in thrombosis induction and progression as previously described. ${ }^{27}$ To the best of our knowledge, reverse remod- 

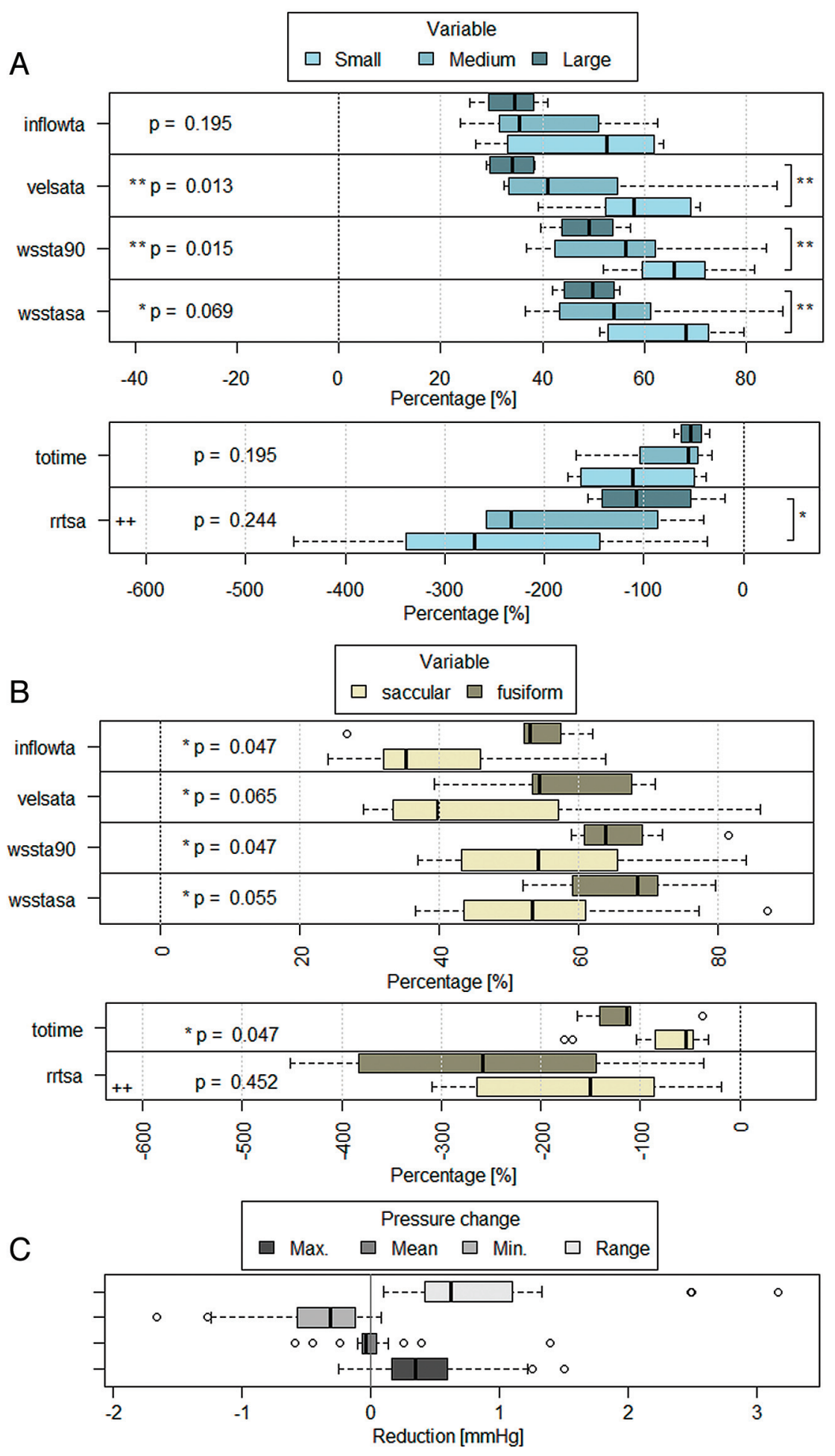

FIG 4. A, Hemodynamic variable reduction by group for the studied hemodynamic variables shown in boxplots classified by aneurysm size. The thick vertical line indicates group median, the box delimits Q1 and Q3, and the dotted lines indicate minimum and maximum. Empty dots indicate outliers ( $>2$ times the interquartile distance from the mean). Double plus signs indicate 2 outliers out of plot range (for rrtsa $=1300 \%$ and $950 \%$, respectively). $P$ values from the corresponding test are presented on the side of each variable group. The asterisk indicates significant to the 10th percentile; double asterisks indicate significant to the 5th percentile. Brackets on the right side show statistical significance between the small and large/giant groups. $B$, Hemodynamic variables classified by aneurysm shape following the same representation as in $A$. Outliers out of range correspond to the same cases as seen in C. Pressure (mean, maximum, minimum, and pressure range) reduction in all aneurysms is expressed in millimeters of mercury. ${ }^{10-13}$ elling in aneurysms treated with an FD is associated with the stagnation of flow inside the aneurysm. The placement of the FD will reduce flow into the aneurysm, increasing turnover time, decreasing WSS in the aneurysm wall, and, most important, near-wall residence time. These changes will, in a successful treatment, promote thrombus formation inside the aneurysm, which will ultimately occupy the whole aneurysm, occluding it and remodelling the parent vessel. Therefore, reverse remodelling is related to WSS, but not only to it. Other variables are involved in the process, some are mechanical and others are biologic. The complete process of vascular remodelling in FD treatment is not yet completely understood.

The reader should be aware that the assumptions made by the models used in this study have some implications on the results.

1) Because only the portion of the FD covering the aneurysm neck is modeled, possible increase in flow resistance in the parent artery induced by the FD might not represent the actual pressure drop in the segment. Still, in this work, we focused on pressure changes inside the aneurysm and not on the parent vessel. The former should not be affected by the use of a portion of the FD.

2) The vascular wall was modeled as rigid, implying that pressure is overestimated. Still, pressure changes (increase/ decrease) due to the implantation of FDs, which are properly captured by the models, are reported.

3) Peripheral bed resistance was not taken into account, and only modeled pressures from a $1 \mathrm{D}$ arterial tree model were considered at the outlets. Because of these assumptions, the magnitude of the variables is an approximation. Nevertheless, the trends observed remain valid and generic because the differences mainly rely on anatomic differences between the cases. For this reason, only relative changes were considered.

Idealized FD deployment was considered in all the cases, which may not always be the case in the clinical setting.

\section{CONCLUSIONS}

Although all hemodynamic variables were significantly reduced after the placement of FDs, no changes were found in 
mean intra-aneurysmal pressure. Maximum pressure was decreased, and minimum pressure was increased; these changes led to an effective reduction of pressure range inside the aneurysm.

When comparing hemodynamic variables (WSS, velocity, and residence time) in the presence of FDs in aneurysms of different sizes, we observed a larger relative reduction in small aneurysms than in large/giant ones. Similarly, larger reductions were observed for fusiform than for saccular aneurysms in terms of high WSS, velocity, and turnover time. Although the hemodynamic variables were always reduced inside the aneurysm after placement of FDs, flow-pattern changes were only observed in a few cases.

The use of CFD provides additional tools for the assessment of IA treatment. As such, they should be used and interpreted with caution, considering the limitations and assumptions of these models.

Disclosures: Ignacio Larrabide-RELATED: Grant: CDTI-Spanish Ministry of Industry, * Support for Travel to Meetings for the Study or Other Purposes: CDTI-Spanish Ministry of Industry, European Commission, ${ }^{*}$ UNRELATED: Employment: CIBER-BBN, Universitat Pompeu Fabra, Grants/Grants Pending: CDTI-Spanish Ministry of Industry, ACCIO, AGAUR (Spain).* Zsolt Kulcsár-UNRELATED: Payment for Lectures (including service on Speakers Bureaus): Penumbra, ${ }^{*}$ Other: Penumbra, ${ }^{*}$ Balt International, ${ }^{*}$ Comments: proctorship. Daniel Rüfenacht—RELATED: Grant: University of Geneva, ${ }^{*}$ Comments: IST-FP6 project @neirulST - Integrated Biomedical Informatics for the Management of Cerebral Aneurysms. Alejandro F. Frangi-RELATED: Grant: CDTI-Spanish Ministry of Economy and Knowledge. *Money paid to the institution.

\section{REFERENCES}

1. Szikora I, Nelson PK, Berentei Z, et al. The potential of flow modification in the treatment of intracranial aneurysms. Interv Neuroradiol 2008;14(suppl 1):77-80

2. Sadasivan C, Cesar L, Seong J, et al. Treatment of rabbit elastaseinduced aneurysm models by flow diverters: development of quantifiable indexes of device performance using digital subtraction angiography. IEEE Trans Med Imaging 2009;28:1117-25

3. Sadasivan C, Lieber BB, Gounis MJ, et al. Angiographic quantification of contrast medium washout from cerebral aneurysms after stent placement. AJNR Am J Neuroradiol 2002;23:1214-21

4. Kallmes DF, Ding YH, Dai D, et al. A new endoluminal, flow-disrupting device for treatment of saccular aneurysms. Stroke 2007;38: 2346-52

5. Cebral JR, Mut F, Raschi M, et al. Aneurysm rupture following treatment with flow-diverting stents: computational hemodynamics analysis of treatment. AJNR Am J Neuroradiol 2010;32:1-7

6. Kulcsár Z, Houdart E, Bonafé A, et al. Intra-aneurysmal thrombosis as a possible cause of delayed aneurysm rupture after flow-diversion treatment. AJNR Am J Neuroradiol 2011;23:20-25

7. van Rooij WJ, Sluzewski M. Perforator infarction after placement of a Pipeline flow-diverting stent for an unruptured A1 aneurysm. AJNR Am J Neuroradiol 2010;31:E43-44

8. Klisch J, Turk A, Turner R, et al. Very late thrombosis of flow-diverting constructs after the treatment of large fusiform posterior circulation aneurysms. AJNR Am J Neuroradiol 2011;32:627-32

9. Turowski B, Macht S, Kulcsár Z, et al. Early fatal hemorrhage after endovascular cerebral aneurysm treatment with a flow diverter (Silk-Stent): do we need to rethink our concepts? Neuroradiology 2011;53:37-41

10. Thevenin D, Seshadhri S, Janiga G. Impact of stents and flow diverters on hemodynamics in idealized aneurysm models. J Biomech Eng 2011;133:071005

11. Hassan T, Ahmed YM, Hassan AA. The adverse effects of flow-diverter stent-like devices on the flow pattern of saccular intracranial aneurysm models: computational fluid dynamics study. Acta Neurochir (Wien) 2011;153;1633-40

12. Tremmel M, Xiang J, Natarajan SK, et al. Alteration of intra-aneurysmal hemodynamics for flow diversion using Enterprise and Vision stents. World Neurosurg 2010;74:306-15

13. Augsburger L, Farhat M, Reymond P, et al. Effect of flow diverter porosity on intraaneurysmal blood flow. Klin Neuroradiol 2009;19: 204-14

14. Villa-Uriol MC, Berti G, Hose DR, et al. @neurIST complex information processing toolchain for the integrated management of cerebral aneurysms. Interface Focus 2011;1:308-19

15. Appanaboyina S, Mut F, Löhner R, et al. Simulation of intracranial aneurysm stenting: techniques and challenges. Comput Methods Appl Mech Eng 2009;198:3567-82

16. Hernandez M, Frangi AF. Non-parametric geodesic active regions: method and evaluation for cerebral aneurysms segmentation in 3DRA and CTA. Med Image Anal 2007;11:224-41

17. Larrabide I, Kim M, Augsburger L, et al. Fast virtual deployment of self-expandable stents: method and in vitro evaluation for intracranial aneurysmal stenting. Med Image Anal 2012;16:721-30

18. Reymond P, Merenda F, Perren F, et al. Validation of a one-dimensional model of the systemic arterial tree. Am J Physiol Heart Circ Physiol 2009;297:H208-22

19. Marzo A, Singh P, Reymond P, et al. Influence of inlet boundary conditions on the local haemodynamics of intracranial aneurysms. Comput Methods Biomech Biomed Engin 2009;12:431-44

20. Bernardini A, Larrabide I, Morales HG, et al. Influence of different computational approaches for stent deployment on cerebral aneurysm haemodynamics. Interface Focus 2011;1:338-48

21. Shojima M, Oshima M, Takagi K, et al. Magnitude and role of wall shear stress on cerebral aneurysm: computational fluid dynamic study of $\mathbf{2 0}$ middle cerebral artery aneurysms. Stroke 2004;35: 2500-05

22. Himburg HA, Grzybowski DM, Hazel AL, et al. Spatial comparison between wall shear stress measures and porcine arterial endothelial permeability. Am J Physiol Heart Circ Physiol 2004;286:H1916-22

23. Wootton $\mathrm{DM}, \mathrm{Ku} \mathrm{DN}$. Fluid mechanics of vascular systems, diseases, and thrombosis. Annu Rev Biomed Eng 1999;1:299-329

24. Schneiders JJ, Vanbavel E, Majoie CB, et al. A flow-diverting stent is not a pressure-diverting stent. AJNR Am J Neuroradiol 2013;34:E1-4

25. Hengl TR: R: A Language and Environment for Statistical Computing. Vienna, Austria; R Foundation for Statistical Computing; 2009

26. Ishibashi T, Murayama Y, Urashima M, et al. Unruptured intracranial aneurysms: incidence of rupture and risk factors. Stroke 2009; 40:313-16

27. Chatzizisis YS, Coskun AU, Jonas M, et al. Role of endothelial shear stress in the natural history of coronary atherosclerosis and vascular remodeling: molecular, cellular, and vascular behavior. $\mathrm{J} \mathrm{Am}$ Coll Cardiol 2007;49:2379-93 